\title{
Cálculo del costo de capital en empresas que no cotizan en la bolsa de valores colombiana
}

\author{
pags 7-19 \\ Grupo de Investigación: Contabilidad Finanzas y Gestión Pública \\ Julio Cesar Millán Solarte• Omar de Jesús Montilla Galvis•• \\ Universidad del Valle
}

Recibido: 12 de febrero de 2014

Aceptado: 30 de mayo de 2014

\section{RESUMEN}

El artículo presenta los resultados del cálculo del Costo de Capital en una compañía que no transa sus acciones en la bolsa de valores de Colombia. Los datos utilizados y la metodología empleada se aplicaron para los índices financieros de los años 2008 y 2009. La técnica utilizada permitió obtener la rentabilidad mínima que debe exigirse a los activos de la compañía estudiada, para devolver las exigencias de retorno que realizan los agentes inversionistas. Se pudo establecer el paralelo entre esta rentabilidad y la rentabilidad de los activos; la rentabilidad del patrimonio y los índices en el orden local, departamental y nacional, para las empresas del mismo sector productivo de la compañía analizada.

Palabras clave: Costo Promedio Ponderado de Capital, Riesgo de Mercado, Índice Beta, ROE, ROA.

\begin{abstract}
This article presents the results in calculating the Cost of Capital in a company that doesn't trade its shares on the stock exchange in Colombia. The used data and the methodology were applied to financial ratios on 2008 and 2009. The used technique permitted to obtain the minimum return demandable of the studied company's assets, to return the profitability demands that the investors agents make. The present study established the parallel between this return and the profitability assets; the return of the patrimony and the indexes at the local, state and national order, for companies in the same productive sector of the analyzed company.
\end{abstract}

Keywords: Weighted Average Cost of Capital, market risk, Beta ratio, ROE, ROA.

\footnotetext{
- Msc,.en Organizaciones Universidad del Valle, Contador Público Universidad del Valle, Especialista en Finanzas Universidad del Valle, Doctorando en Administración - Universidad del Valle. julio.millan@correounivalle.edu.co

- Contador Público, Especialista en Gerencia Financiera con Énfasis Internacional Universidad Libre, Magíster en Administración de Empresas Universidad del Valle, Magister en Gestión Empresarial, Universidad Libre; Candidato a Doctor en Nuevas Tendencias de Dirección de Empresas Universidad de Salamanca. omontill@yahoo.com.mx.
} 


\section{INTRODUCCIÓN}

Toda inversión requiere capital. Por ejemplo, el desembolso inicial para montar un centro de investigación y desarrollar proyectos; el precio de compra de una nueva máquina para su reemplazo; o el desarrollo de una nueva planta de fabricación: todas estas acciones requieren de capital. El flujo de dinero para financiar estos proyectos de inversión es un flujo saliente neto, antes de que exista un conjunto, o set, de flujos de dinero (entradas) generados por la organización. Las fuentes de fondos para prever estos flujos son, en esencia, los propietarios de la empresa (accionistas) y los prestamistas (los titulares de deuda).

La empresa tiene que atraer tanto a inversores que compren acciones de la compañía, como a los prestamistas que inyectan capital. Para hacer esto, la empresa ofrece una rentabilidad a los propietarios y prestamistas: el retorno ofrecido es un "costo" para la compañía, llamado Costo de Capital. Para que un proyecto tenga éxito económico y agregue valor a la empresa, la rentabilidad del proyecto debe, por lo menos, cubrir los costos de obtención. Como resultado, el Costo de Capital representa el rendimiento esperado de todas las actividades de la empresa.

Accionistas o prestamistas reciben de diferentes maneras la recompensa de invertir en la empresa. Tal recompensa para los prestamistas es el pago de un interés por el préstamo, según el tiempo en que se amortice el capital. La recompensa para los propietarios se da en dos formas: 1. los dividendos que se pagan en intervalos regulares de tiempo y 2 . Un incremento del valor de la empresa en el tiempo. El Costo de Capital refleja el costo de recompensar a los propietarios (Costo de Capital) y a los prestamistas (Costo de la Deuda) por su inversión en la compañía.

Entre las herramientas financieras que utiliza la gerencia para llevar a cabo su función, se encuentra el diagnóstico financiero. Éste permi- te visualizar la situación de la empresa y evaluar su desempeño durante un periodo de tiempo. De igual forma ocurre con el Costo de Capital cuya evaluación constituye un parámetro fundamental en la gestión financiera. Esto, en el caso de ser considerado como tasa de descuento, en la evaluación de las proyecciones de los beneficios esperados de nuevos productos o de inversiones. Su determinación errónea conduciría a decisiones perjudiciales, porque las tasas sobrestimadas rechazarían oportunidades rentables; además, frente a un mercado agresivamente competitivo no se debe improvisar, porque conllevaría al deterioro del valor de la compañía.

Esta investigación presenta las técnicas y herramientas financieras utilizadas en el cálculo del Costo de Capital para una empresa que no transa sus acciones en la bolsa de valores. Para tal efecto se han tomado datos reales de una empresa vallecaucana que tiene como actividad comercial la venta de productos y la prestación de servicios, relacionados con el mantenimiento de vehículos. La información utilizada corresponde al periodo 2008 y 2009. Se contemplan varios aspectos, entre ellos, la motivación orientada por el mundo financiero y la importancia que tienen las finanzas en el desarrollo de las organizaciones en un entorno económico. Además, la utilidad y la exigencia de información relevante y útil para proporcionar una base firme en la toma de decisiones.

Este estudio se enfoca en describir la estructura financiera, además, en calcular su Costo de Capital basado en el modelo WACC a través de la aplicación del CAPM. Debido a limitaciones de información de mercado, proporcionada por la compañía, se utilizará la metodología pure play para determinar los coeficientes de riesgo de la empresa con respecto al mercado.

Dentro de una entidad empresarial existen varios criterios para tomar decisiones y, en la mayoría de los casos, estas dependen de la cantidad de información que se tenga disponible. Las decisiones vitales para la empresa van en- 
LÍNEA DE INVESTIGACIÓN: MEDICIÓN DEL RIESGO DE CRÉDITO Y LIQUIDEZ

caminadas al crecimiento y posicionamiento en el mercado. Por esta razón siempre dependen de información de su entorno (clientes y competidores) para tomar decisiones con base en esta información y mantener su crecimiento.

Cuando la organización atraviesa por una crisis, ésta no es la única información que se desea observar. Se revisan otras fuentes, como es el caso de los gastos en que se incurre, para poder dar alivio a la utilidad, de manera que se genere rentabilidad y así se asegure su crecimiento. En ciertos casos cuando esto no compensa, se promueve por la consecución de otras salidas las cuales son desconocidas, en otros casos, por pequeñas y medianas empresas. Una de ellas es la valoración de su capital, que consiste en dar a conocer si la comercialización o prestación de servicios es una actividad que genera valor para ella y para sus dueños.

Para la obtención del Costo Promedio Ponderado de Capital en la compañía estudiada, debe tenerse en cuenta varios factores del mercado que representan un riesgo para ella, entre estos:

- Devaluación.

- Comercialización de baterías recicladas y llantas usadas.

- Inseguridad en las carreteras nacionales, lo que reduce el mantenimiento y circulación de vehículos.

- Baja demanda por la crisis económica.

- Grandes cadenas de almacenes: por competencia de precios.

Pero también deben tenerse en cuenta las fortalezas del mercado que deben ser aprovechadas por la empresa. Entre ellas están:

- Productos básicos de mantenimiento para el vehículo.
- Amplio portafolio de productos.

- Incremento del parque automotor.

- Convenios con proveedores, dirigidos a la promoción y publicidad del producto y el establecimiento.

Vélez Pareja (2002), plantea diferencias en Costo de Capital y la forma actual de su determinación, para firmas no transadas en bolsa. Menciona que, estadísticamente, el método más utilizado en la actualidad es el CAPM (Modelo de Valoración de Activos de Capital), el cual fue creado para aplicarse fundamentalmente a empresas que cotizan en bolsa, porque estas poseen teóricamente toda la información histórica para aplicar a plenitud el modelo. El análisis muestra que para el año 2000 el número de firmas existentes en Estados Unidos, era de 5.607.743. Teniendo en cuenta que en el año 2002, la cifra de empresas inscritas en bolsa ascendía a 7510 , se puede tener una relación entre estos datos, que si bien pertenecen a periodos diferentes, presentan una ilustración aproximada de la participación que tenían las firmas en dicho país en su mercado bursátil.

Martínez Arias, (2006) da una breve explicación del Costo de Capital y su composición: plantea de manera muy específica, y a través de ilustraciones matemáticas, modelos como el de valuación de dividendos y el CAPM; también diseña explicaciones sobre el riesgo sistemático o de mercado, y una manera de hallar el Beta (volatilidad de las acciones).

Ehrhardt y Brigham, (2007) evalúan los conceptos fundamentales de finanzas corporativas, tipos de riesgos y rendimiento. Además de los proyectos y su valuación, donde describen el Costo de Capital con cada uno de sus componentes, explican la metodología de reconocido valor técnico, aplicada en nuestros días. También muestran la importancia de las decisiones con base en la estructura de capital. 
Kerins, Janet Kiholm Smith, Richard Smith Source (2003), realizan el estudio Opportunity cost of capital for venture capital investors and entrepreneurs donde estiman el Costo de Capital para que los inversionistas y empresarios se aventuren en esta clase de negocios. En las empresas de fase temprana, estiman los efectos de la diversificación, industrialización, y madurez financiera en el costo de oportunidad, asumiendo un periodo de tendencia de un año. Concluyen que el costo de oportunidad de un empresario es de dos a cuatro veces más alto que el de un inversionista bien diversificado.

Domínguez (2007), se enfoca básicamente en la medición de los rendimientos que pueden generar proyectos de inversión a través de estudios econométricos del Costo de Capital. La metodología usada se basa en la teoría de asignación de precios de activos para modelar la tasa esperada en Brasil, Colombia, México y Perú para finales de 2006. Altuve (2006) explica en forma simplificada el Costo de Capital, planteándolo como una herramienta vinculada a la forma de financiamiento de cualquier tipo de organización, debido a que encierra todos sus aspectos de financiación. La metodología empleada es la del CPPC (Costo Promedio Ponderado de Capital). Luego genera un desarrollo más técnico acerca de lo que en su perspectiva es el Costo de Capital, expresando que es aquella tasa que iguala el valor actual de los fondos realmente percibidos por la empresa, con el valor actual de los egresos que provocará la operación final. Para terminar, ratifica la posición con respecto a la financiación de las PYMES, porque estas poseen unos costos de financiación más elevados que las empresas grandes por poseer un grado de riesgo mayor. Por tal razón es conveniente planear de manera objetiva, y basada en ciertos análisis, la financiación que se realice a través de terceros, y no como el resultado de malas gestiones operativas o comerciales de la empresa.

Herrera y Mora (1998) realizaron un estudio acerca del mercado de capitales en el ámbito nacional, el cual resultó en ciertas tendencias que se descubrieron a partir de una muestra realizada a un sector de la economía colombiana. Se pudo comprobar que la forma de financiación en las organizaciones colombianas, se encontraba en un proceso evolutivo, debido a que las compañías se enfocaban en adquirir recursos bajo la figura de los préstamos como medio de financiación, quitándole terreno a la porción de capital propio. Lo cual trae a colación el tema central: la tasa de retorno que el financiador de un proyecto puede obtener, opta por realizar una inversión y no otra con un rango económico equivalente, es decir el Costo de Capital.

\section{MATERIALES Y MÉTODO}

\section{Datos y fuentes de información}

Una forma de establecer relaciones de índole financiero es generando comparaciones con organizaciones presentes en el mercado, dedicadas a la misma actividad. Por esta razón se analizaron varios indicadores obtenidos a través de la Superintendencia de Sociedades, con información de empresas del mismo sector económico. La compañía estudiada conserva un buen margen bruto porque supera en gran proporción al mercado nacional, departamental y municipal. Para el año 2009 su liquidez se encuentra dentro de parámetros normales, de acuerdo a la tendencia del mercado. Respecto del endeudamiento, se puede decir que es comparativamente alto. Sin embargo, posee una ventaja debido a que su endeudamiento, en relación con el mercado, no se concentra en el corto plazo como es el caso de la mayoría de las empresas de este sector en la ciudad de Santiago Cali. Los demás datos necesarios para llevar a cabo la investigación fueron proporcionados por la compañía estudiada e investigaciones sobre el tema.

\section{Estructura financiera}

La estructura de capital hace referencia a las proporciones que guardan la deuda y las accio- 
LÍNEA DE INVESTIGACIÓN: MEDICIÓN DEL RIESGO DE CRÉDITO Y LIQUIDEZ

nes en el balance. La estructura de capital hace énfasis en las porciones de financiamiento a largo plazo. Para reconocer un financiamiento que haga parte de la estructura de capital debe cumplir las siguientes características:

\section{Costos}

La estructura financiera es la forma como se financian los activos de una empresa. Se debe analizar el concepto de riesgo de los dos componentes que constituyen la financiación de la empresa. El Patrimonio representa mayor grado de riesgo que el Pasivo. Modigliani y Miller (1958), afirmaron que no existe una estructura óptima de capital, por ende, las decisiones de financiación no influyen en el valor de las empresas. Tal afirmación se basa en supuestos, como el de mercados de capitales perfectos y sin impuestos.

Los autores aclaran que cuando se aterrizan en la práctica los conceptos teóricos, se encuentran con las imperfecciones del mercado, las cuales brindan la posibilidad de masificar el valor de las empresas a través de una combinación óptima de financiación, que considere el equilibrio entre los costos de deuda y los beneficios generados por las mismas.

En los años sesenta la teoría de portafolios, esbozada por Harry Markowitz y complementado por James Tobin, trata esencialmente de una serie de combinaciones a realizar con títulos financieros, con el fin de disminuir el riesgo no diversificable o sistemático (Riesgo de mercado).

A partir de la teoría de portafolios, nace la base de estas investigaciones, el Modelo de Valoración de Activos de Capital (CAPM), desarrollado por William F. Sharpe y John Lintner, donde se expone que el rendimiento esperado de un activo en equilibrio, debe ser correspondiente a su riesgo sistemático.

La tasa de descuento conocida como Costo de Capital (aproximación del costo de oportunidad de quienes proveen los recursos), es una tasa de retorno que el financiador de un proyecto puede tener, si opta por realizar esa y no otra inversión con un riesgo equivalente. Tal porcentaje es una base decisoria sobre el rumbo de las inversiones, la cual obedece específicamente al riesgo de mercado, y a las oportunidades de inversión.

La metodología más empleada para hallar el Costo de Capital, es el promedio ponderado de tal costo, más conocido por sus siglas en inglés como WACC ("Weighted Average Cost of Capital) en Modigliani y Miller (1958), es una herramienta que permite ponderar la participación (Estructura de capital) de las fuentes y sus correspondientes costos, en una sola ecuación que permite hallar el costo total del capital en la empresa objeto de estudio.

$$
W A C C=\left(P d^{*} K d\right)+\left(P e^{*} K e\right)
$$

Dónde:
$\mathrm{Pd}=$ Porcentaje de las inversiones financiadas con deuda.

$\mathrm{Kd}=$ Costo de deuda

$\mathrm{Pe}=$ Porcentaje de las inversiones financiadas con el capital propio.

$\mathrm{Ke}=$ Costo de Capital propio.

\section{Costo de Deuda}

Es la tasa de Interés que se cobra a las empresas, por los recursos entregados para apalancar sus Inversiones, en relación con el cálculo del costo exigido por los accionistas al endeudamiento $(\mathrm{kd})$. Es relativamente más fácil de obtener, porque surge de dividir los intereses pagados (más los costos de emisión de la deuda), por el valor en libros de la deuda. Este resultado brinda la tasa que efectivamente paga la empresa por la deuda contraída.

$W A C C=P d^{*} K d(1-t)+\left(P e^{*} K e\right)$ 
Dónde:

$P d=$ Porcentaje de las inversiones financiadas con deuda.

$\mathrm{Kd}=$ Costo de deuda.

$\mathrm{Pe}=$ Porcentaje de las inversiones financiadas con el capital propio.

$\mathrm{Ke}=$ Costo de Capital propio.

$\mathrm{t}=$ Tasa porcentual del impuesto a la renta.

Costo de Capital Propio

Antes de empezar a hablar sobre el valor del capital propio, se debe tener en cuenta el factor riesgo, el cual se cataloga como una diferencia notable a la hora de comparar las fuentes de financiamiento. La deuda tiene menos riesgo que el patrimonio, por lo tanto, en condiciones normales, siempre será menor el costo porcentual de la deuda que el del patrimonio.

Partiendo de esta premisa podemos entrar a definir el Costo del Capital propio como el valor porcentual que representa el riesgo asumido por los dueños del capital, en una organización determinada. Esta tasa requiere de ciertos modelos para su cálculo ya que está cargada de mayor complejidad que la anterior. Dentro de los modelos más utilizados están:

- Modelo de Capital Asset Pricing Model (CAPM) y APT ${ }^{1}$ para mercados perfectamente competitivos

- Modelos para medir activos que no cuentan con mercados. Modelo de similares o Pure Play

1 APT (El arbitraje Pricing Theory): metodología utilizada para la medición del costo de capital en mercados perfectamente competitivos.

\section{Modelo CAPM (Capital Asset Pricing Model)}

Según Vélez Pareja (2002) en el CAPM “La rentabilidad de una acción está relacionada en forma lineal con la tasa libre de riesgo de una economía y con la rentabilidad del mercado de acciones como un todo". El CAPM muestra que el Costo de Capital propio de un activo es igual al retorno proveniente de un activo libre de riesgo, más la prima por el riesgo asumido, la cual es igual al valor del Beta (el cociente entre la covarianza del retorno del activo y el retorno del mercado, con la varianza del retorno del mercado) por la prima de riesgo del mercado)

El modelo establece que el costo del patrimonio es igual al retorno de un activo libre de riesgo, más una prima de riesgo relacionada con el sector de desempeño de la empresa, así:

$K e=R f+P r$

Dónde:

$\mathrm{Rf}=$ Es el retorno de un activo libre de riesgo.

$\operatorname{Pr}=$ Prima de riesgo.

La prima de riesgo mide el rendimiento adicional que un inversor requiere, para mantener una cartera diversificada de acciones en lugar de un activo libre de riesgo.

$K e=R f+\mathrm{b}(R m-R f)$

Dónde:

Ke: $\quad$ Rentabilidad de la acción

Rf: $\quad$ Prima libre de riesgo

$\beta$ : $\quad$ Múltiplo o Beta

$(\mathrm{Rm}-\mathrm{Rf})$ : Prima de riesgo de mercado. 
LÍNEA DE INVESTIGACIÓN: MEDICIÓN DEL RIESGO DE CRÉDITO Y LIQUIDEZ

La proporcionalidad existente entre la prima de riesgo de la empresa, y la del mercado, es conocida como un coeficiente de proporcionalidad o coeficiente Beta, la cual mide la sensibilidad de la rentabilidad en la organización, con las variaciones del mercado.

\section{Coeficiente Beta}

El coeficiente Beta representa la rentabilidad de la empresa con respecto a la rentabilidad del mercado. La definición real del Beta es la covarianza entre la rentabilidad del mercado y la rentabilidad del sector con respecto a la economía en general, divido por la varianza del mercado

El Beta es un indicador que mide la sensibilidad (cambio promedio en porcentaje), de los riesgos de una compañía (Acción, proyecto, etc.), con respecto a los movimientos del mercado. El coeficiente Beta indica la respuesta del rendimiento de una acción ante el riesgo sistemático:

"El coeficiente Beta de las acciones de una empresa aumenta linealmente a medida que aumenta el apalancamiento financiero de una empresa (sic). Además de estos existen muchos otros factores que pueden afectar al Beta, como son la razón de pago de dividendos, la liquidez, el tamaño de la empresa, la tasa de crecimiento, etc.". (Weston y Copeland, 1995, p 477).

La expresión para hallar el valor $\beta$ es:

$$
\beta i=\frac{\operatorname{COV}(R i, R m)}{\sigma_{m}^{2}}
$$

Dónde:

$$
\begin{array}{ll}
\beta i= & \text { Beta del Activo i } \\
\operatorname{Cov}(\mathrm{Ri}, \mathrm{Rm})= & \begin{array}{l}
\text { Covarianza entre el riesgo del } \\
\text { activo i y el riesgo de mercado }
\end{array} \\
\sigma_{m}^{2}= & \text { Varianza del mercado }
\end{array}
$$

Con respecto a la premisa del CAPM en la cual se relaciona la rentabilidad versus el riesgo, se puede decir que a mayor rentabilidad obtenida por el inversionista, acarreará asumir un riesgo mayor, de tal forma que se puede analizar de manera más comprensible los siguientes valores de Beta:

\section{Tabla 1. Interpretación del BETA}

\begin{tabular}{|l|l|}
\hline$\beta<1$ & $\begin{array}{l}\text { El riesgo de una beta inferior a uno es me- } \\
\text { nor, lo que haría pensar que se adoptó una } \\
\text { medida conservadora en términos del ries- } \\
\text { go asumido. }\end{array}$ \\
\hline$\beta=1$ & $\begin{array}{l}\text { Beta igual a uno sugiere que la inversión } \\
\text { debe moverse al ritmo del mercado. }\end{array}$ \\
\hline$\beta>1$ & $\begin{array}{l}\text { La sensibilidad de la inversión es mayor } \\
\text { a la del mercado, por ende el riesgo de la } \\
\text { misma tiende a ser superior al mercado. }\end{array}$ \\
\hline
\end{tabular}

Hay una gran dificultad en Colombia para determinar de manera directa el Beta, que refleje el riesgo del sector al cual pertenecen las empresas analizadas, ya que las que operan específicamente en tal sector, y que cotizan en la bolsa colombiana, son escasas, lo cual imposibilita encontrar un riesgo de mercado confiable.

El retorno contable se puede medir a partir de la utilidad contable (operativa, o neta), como rendimiento contable sobre el patrimonio $(\mathrm{ROE})^{2}$, el cual resulta ser un indicador que relaciona la utilidad contable sobre el mismo; el ROA (Retorno sobre Activos), otra medida análoga, puede ser un índice de bolsa, o un índice de mercado (ROE O ROA promedio de mercado).

Un método que busca subsanar el inconveniente que tiene la compañía estudiada, para

2 ROE: Representa el porcentaje anualizado de ganancia, obtenido por la empresa, en relación a la inversión anualizada de los accionistas. Como la utilidad ya lleva el efecto de actualizaciones en costo y depreciación, así como el resultado por efecto de actualizaciones en costo y depreciación, así como el resultado por posición monetaria y se encuentra en pesos de poder adquisitivo de la fecha de cierre, la tasa obtenida será una tasa real. 
calcular su Beta, es conocido como Puré play o método de similares. Este método supone que el Beta de un activo transado en el mercado es un dato estadístico suficiente para calcular su rendimiento requerido de equilibrio.

La compañía estudiada representa la realidad colombiana en el tema bursátil ya que, en él, la gran mayoría de empresas colombianas no se tranzan en la bolsa de valores, por tanto, no se poseen datos estadísticos que provean de información necesaria para la determinación del Beta. Para tales casos el Pure Play ofrece una alternativa encaminada a reconocer la existencia paralela de activos o compañías (en líneas de negocios similares) que se negocian efectivamente en el mercado. Lo que se hace es observar el rendimiento requerido del equilibrio de un activo similar. Con esta información se puede aproximar el retorno requerido (Costo del Capital Accionario) del activo cuyo riesgo queremos evaluar.

El método consiste en elegir una empresa (o grupo de empresas), en el mercado de referencia de la investigación (EEUU), que tenga características similares a la compañía estudiada. Cuando se habla de características similares, se hace referencia básicamente a:

- La empresa o grupos de empresas deben poseer una actividad económica o pertenecer a un sector económico correspondiente, es decir que se relacione con el sector al cual pertenece la compañía analizada, y además con la comercialización de llantas para automotores.

- El proyecto, la división, o la compañía analizada se encuentra en una línea de producto bien definida.

- La estructura de costos fijos y variables es similar

- Cuando se identifica más de una compañía como un potencial comparable, suele tomar- se la mediana del Beta de las compañías identificadas.

- Regularmente el coeficiente obtenido de la comparada(s), posee una estructura de capital diferente a la de la compañía estudiada, debe entonces des-apalancarse ${ }^{3}$ el Beta, para ajustarla a la estructura original de la compañía estudiada.

Cuando se desea aplicar un método de similares (Betas comparadas), se tiene en cuenta solo el riesgo fundamental u operacional del negocio, el cual se mide a través del Beta desapalancado o Beta del activo (Ba). Sin considerar la presencia de impuestos, el Ba se obtiene a partir de la siguiente fórmula:

$\beta a=\beta \frac{K P}{V}+\beta d \frac{D}{V}$

Dónde:

$\beta d$ es el Beta de la deuda de la acción.

Cuando se introduce el impuesto a la fórmula se tendría:

Para des-apalancar $\quad \beta a=b /(1+(1-t) D / K P(7)$

Para Apalancar $\quad \beta l=\beta a /(1+(1-t) D / K P(8)$

Dónde:

$\beta a$ Beta desapalancada u operativo

Bl Beta apalancada o Beta del patrimonio.

En resumen, se toma el Beta de la similar aplicando la fórmula (7), se le aparta el factor de apalancamiento contraído en el medio donde se desarrolla. Con la fórmula (8) se apalanca de acuerdo a las circunstancias de la estructura de la compañía estudiada.

3 Des-apalancar Consiste en apartar del coeficientes Beta, (extraído de la empresa modelo), el riesgo financiero y la tasa de impuestos de tal empresa comparada. 
LÍNEA DE INVESTIGACIÓN: MEDICIÓN DEL RIESGO DE CRÉDITO Y LIQUIDEZ

La metodología que se utiliza en este trabajo para hallar el riesgo de mercado es básicamente la planteada por García (2003) donde indica que "la diferencia entre los promedios históricos y la rentabilidad de los bonos del Tesoro".

Por esta razón se tomará (para determinar el riesgo de mercado en cifras históricas en el caso de la compañía estudiada) los datos de la reserva federal de los Estados Unidos, los cuales poseen un compendio de rendimientos otorgados usualmente por las acciones y los bonos del estado desde 1928 hasta la fecha. Específicamente se utiliza un promedio aritmético del spread del rendimiento del S\&P500 sobre la tasa libre de riesgo lo cual arroja el valor promedio del riesgo de mercado.

La mayoría de las empresas presentes en nuestro país son organizaciones que no se tranzan en la bolsa de valores. La información histórica y de mercado que poseen es muy limitada, lo cual hace necesario la aplicación de técnicas alternas que propenden por acercarse a la realidad económica de dichas compañías, a los valores que puedan ser reconocidos para tomar decisiones dentro del mercado.

La compañía estudiada actualmente está muy cerca de su estructura óptima de capital, si se mantiene la proporción en el valor cobrado por terceros, y se cuente con una capacidad plena tanto en rentabilidad como en liquidez, para cubrir, sin incurrir en costos extras, el valor equivalente al gran endeudamiento que posee la entidad.

Es de notar que en condiciones normales muchas personas afirman que el endeudamiento a través de terceros es realmente menos costoso en relación al capital propio. Si bien pueden tener razón en parte, no siempre se puede reconocer esta afirmación como cierta, ya que existe un punto de inflexión, en el cual el rendimiento exigido por la deuda se ubica en niveles superiores a los reclamados por el capital propio. Dicho punto hace referencia al riesgo, es decir: cuando la empresa sobrepasa niveles de endeudamiento, hacen que esta vía de financiamiento incremente su nivel de riesgo lo cual genera que dichos terceros, exijan un rendimiento superior a los socios del ente económico.

\section{RESULTADOS Y DISCUSIÓN}

Una vez analizada la información provista en los estados financieros se obtiene lo siguiente:

Tabla 2. Datos de la Organización

\begin{tabular}{|l|c|c|c|}
\hline \multicolumn{1}{|c|}{ Detalle } & Año 2008 & Año 2009 & Promedio \\
\hline Activo & $\$ 2.213 .319 .924$ & $\$ 2.166 .640 .411$ & $\$ 2.189 .980 .167$ \\
\hline Activo Corriente & 767.086 .081 & 581.585 .586 & 674.335 .834 \\
\hline Clientes & 569.744 .860 & 722.240 .406 & 690.992 .633 \\
\hline Inventarios & 423.848 .648 & 395.065 .267 & 409.456 .958 \\
\hline Pasivo & 1.861 .799 .770 & 1.812 .285 .918 & 1.837 .042 .844 \\
\hline Pasivo Corriente & 1.308 .474 .875 & 1.330 .262 .559 & 1.319 .368 .717 \\
\hline Proveedores Nacionales & 665.833 .511 & 864.122 .413 & 764.977 .962 \\
\hline Patrimonio & 351.520 .154 & 354.354 .493 & 352.937 .324 \\
\hline Ingresos Neto & 4.935 .268 .564 & 4.697 .398 .047 & 4.816 .333 .306 \\
\hline Margen Ebitda & $1,18 \%$ & $2,99 \%$ & 2,59 \\
\hline Depreciación & 127.777 .138 & 121.683 .848 & 124.730 .493 \\
\hline Gastos Financieros (Intereses) & 161.475 .492 & 6.622 .351 & 17.783 .922 \\
\hline Impuesto de Renta & $33 \%$ & $33 \%$ & $33 \%$ \\
\hline
\end{tabular}

Fuente: Calculo de los autores con base en Estados Financiero 
Se puede observar la diferencia entre los índices contables ROA y ROE para la empresa y los correspondientes al sector en el orden territorial. Se pudo calcular información relevante que permite establecer relación entre los índices empresariales y el sector, tal como se muestra a continuación:

Tabla 3.

\begin{tabular}{|l|l|c|c|c|c|c|}
\hline \multirow{2}{*}{ Indicador } & \multicolumn{1}{|c|}{ Descripción } & $\begin{array}{c}\text { Promedio 2008-2009 } \\
\text { Compañía } \\
\text { estudiada }\end{array}$ & $\begin{array}{l}\text { Costo } \\
\text { Capital }\end{array}$ & Nacional & Departamental & Municipal \\
\hline \multirow{2}{*}{ ROE } & Utilidad Neta & $5.04 \%$ & $6,13 \%$ & $10,86 \%$ & 3,86 & $6,86 \%$ \\
\cline { 2 - 7 } & Patrimonio & & & & & \\
\hline \multirow{2}{*}{ ROA } & $\begin{array}{l}\text { Utilidad Neta + } \\
\text { (Intereses * (1-t)) }\end{array}$ & $5,18 \%$ & $6,13 \%$ & $\begin{array}{l}\text { No se tiene } \\
\text { el valor neto } \\
\text { pagado por } \\
\text { interés }\end{array}$ & $\begin{array}{l}\text { No se tiene } \\
\text { el valor neto } \\
\text { pagado por } \\
\text { intereses }\end{array}$ & $\begin{array}{l}\text { No se tiene } \\
\text { el valor neto } \\
\text { pagado por } \\
\text { interés }\end{array}$ \\
\hline
\end{tabular}

Tabla 4

\begin{tabular}{|l|c|}
\hline Participación deuda & $83,9 \%$ \\
\hline Costo de deuda & $5,20 \%$ \\
\hline Participación patrimonio & $16,1 \%$ \\
\hline Costo del patrimonio en US\$ corrientes & $7,33 \%$ \\
\hline Costo del patrimonio en \$ corrientes & $10,96 \%$ \\
\hline Costo de capital (wacc) & $6,13 \%$ \\
\hline
\end{tabular}

Fuente: Calculo de los autores con base en Estados Financieros

Como se observa en la tabla 1 actualmente la compañía estudiada, posee un costo de deuda equivalente al 5,20\% (una vez aplicado el escudo fiscal). Su Costo de Capital propio hace referencia a $10,96 \%$, teniendo en cuenta la combinación de fuentes $(83,88 \%$ deuda y $16,12 \%$ capital propio), se obtiene un costo promedio ponderado de capital de $6,13 \%$.

Aplicando el método de ensayo y error en la estructura financiera, se llegó a la conclusión de que la manera más indicada para combinar las fuentes en cuestión es de $87 \%$ de deuda y $13 \%$ de capital propio. Suponiendo que el incremento proporcional exigido por otros terceros sea de $0,35 \%$, es decir que pase de $7,7 \%$ a $8,05 \%$, (tal dato se extrajo con base en proporciones). El Costo de Capital propio estaría en un $11 \%$, y el capital de terceros valdría $5,40 \%$, resultando un costo promedio ponderado de capital de $6,12 \%$.

\section{Tabla 5. Estructura financiera óptima}

\begin{tabular}{|l|c|}
\hline Participación deuda & $87,0 \%$ \\
\hline Costo de deuda & $5,40 \%$ \\
\hline Participación patrimonio & $13,0 \%$ \\
\hline Costo del patrimonio en US\$ corrientes & $7,36 \%$ \\
\hline Costo del patrimonio en \$ corrientes & $11,00 \%$ \\
\hline Costo de capital (wacc) & $6,12 \%$ \\
\hline
\end{tabular}

Fuente: Calculo de los autores.

El costo que asume la compañía estudiada, por la financiación de sus activos brutos anualmente en el año 2008 y 2009 equivale al $6.13 \%$ de dichos bienes:

El Costo de Capital refleja el rendimiento de la deuda contraída por la empresa, en aras de cumplir su objeto social. Lo anterior, teniendo en cuenta que la financiación de un ente económico consta de dos componentes, representados por: en primera instancia, por el aporte 
LÍNEA DE INVESTIGACIÓN: MEDICIÓN DEL RIESGO DE CRÉDITO Y LIQUIDEZ

de los socios o dueños de la organización y, en segunda instancia, por terceros que contribuyen con capital: si bien no participan como dueños (es decir no son participes en la decisiones organizacionales), sí ostentan cierto derecho, el cual se ve reflejado en la retribución económica que debe hacer la empresa para cumplir con rendimientos establecidos en títulos valores anteriormente firmados. Por esta razón se entra a revisar en detalle estas diferentes ópticas, y su significado para la compañía estudiada.

- El Costo del Capital propio haciende a $10.96 \%$, el cual supera en $5.75 \%$ el rendimiento exigido por terceros que también financian la empresa.

El riesgo incrementa el valor del capital. Se demuestra que el rol adquirido legalmente por los dueños de la empresa, como responsables y garantes de las operaciones de la misma, debe exigir, en condiciones normales, un rendimiento superior al perseguido por los diferentes terceros. Si bien estos últimos incurren en un grado de riesgo determinado, va a ser solo, por el monto de su inversión, además, en el escalafón de prioridades en caso de una eventual liquidación. Estos están privilegiados jerárquicamente en cierta forma, a la hora de reclamar el compensatorio correspondiente por la inversión efectuada.

- $10.96 \%$ es la tasa de oportunidad que auspician los garantes de la empresa ante una inversión que posea unas características de riesgo similares.

Es necesario aclarar: si los dueños consideran teóricamente cambiar la inversión efectuada en esta organización, deberían conseguir una fuente que les genere una rentabilidad superior a $10,96 \%$ del total invertido. Así tener un nivel de riesgo igual o inferior al presentado por la compañía estudiada, en aras de multiplicar el valor económico que poseen actualmente.

El Costo del Capital efectivamente pagado anualmente a terceros es del $7,77 \%$. Claro está que dicha vía de financiación tiende a disminuir proporcionalmente según la tasa impositiva, una vez aplicado el escudo fiscal, convirtiéndose en un $5,20 \%$ :

Se debe tener en cuenta que el gobierno, a través del Impuesto de Renta, promueve un estilo de auxilio, por llamarlo de alguna manera, al pago que se efectúa por intereses, ya que permite ser descontado de la base gravable de dicho impuesto. El rendimiento exigido por aquellos terceros externos que financian la organización, es proporcional al impuesto (33\% en este caso); así convierte el estilo de financiación, a través de terceros, en la forma menos costosa, siempre y cuando, el riesgo financiero no incremente dicho pago en niveles superiores a los establecidos por el capital propio.

Por otro lado, el $6.13 \%$ debe ser la tasa mínima de retorno que debe exigir la organización como base, en sus actividades de inversión, ya que a partir de dicha cifra cualquier ingreso marginal contribuiría a generar valor económico para la empresa.

Partiendo de esta premisa podemos entrar a definir el Costo del Capital propio como el valor porcentual que representa el riesgo asumido por los dueños del capital. Es decir, en una organización determinada, cualquier inversión ejercida por la compañía estudiada debe exigir como mínimo una contraprestación del 6,13\% anual de su valor. De esta forma se garantizaría que tal rubro, propenda por la generación de valor económico para el ente económico.

\section{CONCLUSIONES}

Se nota claramente que, según los libros contables, la compañía analizada retorna a los socios un $5,04 \%$ del total invertido, porcentaje que no cumple ni siquiera con el promedio ponderado de la inversión. Si se tiene en cuenta que el costo que debería retornar la compañía analizada a sus propietarios es de $10,96 \%$, actualmente tiene una razón de cumplimiento del $54 \%$. 
Por otra parte es de advertir que la inversión realizada en los activos de la compañía analizada no compensa el costo de oportunidad que exige su inversión, ya que el ROA alcanza un $5,18 \%$. Esto significa casi un $0,95 \%$ por debajo de la rentabilidad mínima exigida por los aportantes de capital.

El retorno que la organización ofrece a sus propietarios es inferior al ofrecido a nivel nacional y municipal en $5,82 \%$ y $1,82 \%$ respectivamente. Sin embargo, es mayor al nivel de retorno del patrimonio entregado a nivel departamental, en un $1,18 \%$. Todo lo cual muestra una relativa homogeneidad en cuanto a la zona; este resultado inferior frente al mercado nacional, puede obedecer a que existe una competencia ardua en relación al parque automotor demandante, además el mercado de partes que acaparan los grandes almacenes de cadena que no corresponden al sector genera los retornos inferiores en dichas organizaciones.

El rendimiento que genere la empresa deberá ser suficiente para atraer a los inversores a poner su dinero en la empresa. Si la empresa no proporciona los beneficios requeridos, el valor de su inversión en la compañía disminuirá, ocasionando el encarecimiento de la consecución de fondos en el futuro. Como resultado los administradores de la compañía tienen la responsabilidad de asegurar que ésta retorne la rentabilidad ofrecida y esperada, en los proyectos que emprenda, y que se evalúan contra los retornos esperados tomándolos como punto de referencia.

Dado el contexto de la tasa de descuento que los gerentes financieros utilizan en el control de empresa a través de la elección de proyectos, es importante que haya un método conocido y aplicativo para determinar el Costo de Capital, como se pudo constatar el costo de la deuda es más fácil de determinar, dado que los prestamistas de la empresa requieren sus ganancias en el tipo de interés cobrado, esto significa que el costo de la deuda es la tasa de interés pagada por la empresa para los tenedores de la misma. El Costo de Capital es más difícil de determinar para los socios de la compañía es decir para el patrimonio, ya que los accionistas, esperan sus ganancias en dos formas: como un dividendo ó como un aumento en el valor de su participación en la empresa.

El método más común para determinar el Costo de Capital de la empresa es emplear el Capital Asset Pricing Model, CAPM. Este modelo relaciona la rentabilidad exigida por los inversionistas a la tasa libre de riesgo obtenido en título del gobierno o bonos, y el riesgo de la empresa en relación con el riesgo del mercado.

El Costo de Capital y el costo de la deuda se combinan en un promedio ponderado.

\section{REFERENCIAS}

Aguilera, J. "Costo del capital." Comisión de regulación de agua potable y saneamiento básico - cra -

Blank, L. (1990) "La administración de las organizaciones: un enfoque estratégico." Cali, p. 80.
Brealey, R., Myers, S. (1996). "Principles of corporate finance", Mc. Graw-hill, fifth edition p. 213. U.S.A

Briones, G. (2001) "Métodos y técnicas de la investigación." 2. Ed. Editorial Trillas. México.

Copeland, T., Koller, T., Murrin, J. "Valoración medi- ción y gestión del valor." $3^{a}$. Ed. Ediciones Deusto, Planeta de Agostini profesional y formación. España.

Chisari, O., Rodríguez, P., Martin, A., Rossi S, Martin, A., (1999) "El costo de capital en empresas reguladas: incentivos y metodología." Desarrollo económico. Vol. 38, no. 152, pp. 953-984. Disponible 
LÍNEA DE INVESTIGACIÓN: MEDICIÓN DEL RIESGO DE CRÉDITO Y LIQUIDEZ

en: Http://www.jstor.org/stable/3467264

Dumrauf, G. (2006) "Finanzas corporativas." 3. Ed. AlfaOmega grupo editor s.a. De cv, p. 704. México.

Ehrhardt, M., Brigham, E. (2007) "Finanzas corporativas 2. Ed. International Thomson editores s.a. De c.v. p. 276, México.

Herrera A., Santiago, M., "El costo de capital en las empresas colombianas y el efecto de la tributación". Superintendencia de Valores de Colombia. Santafé de bogotá. P.111, Colombia.
Martínez, C., (2006) "Fundamentación en finanzas". $1 a$. Ed. Editorial Universidad Pontificia Bolivariana, p. 177, Medellín, Colombia.

Proaño, B., Salgado, J. (2005) "Propuesta Metodológica de valoración de empresas aplicada a grandes empresas en el ecuador adaptación de los modelos z'de altman y flujos de caja descontados" Universidad del Azuay Cuenca, $p$. 19, Ecuador.

Rivera, J. (2004) "Introducción a la administración financiera". 1a. Ed. Cali: universidad del valle, p. 307, Colombia.
Rivera, J. (2001) "Costo de capital y estructura financiera para empresas multinacionales. Pliegos administrativos y financieros." Facultad de Ciencias de la Administración. N. 31.

Vélez, I. (2002) "Costo de capital para firmas no transadas en bolsa." 29. Ed. Revista Latinoamericana de Administración, p. 45.

Vélez, I., Tham, J. (2002) "La medición del valor y del costo de capital de la empresa. Monografía de administración, Universidad de los Andes, Serie Finanzas. p. 102 